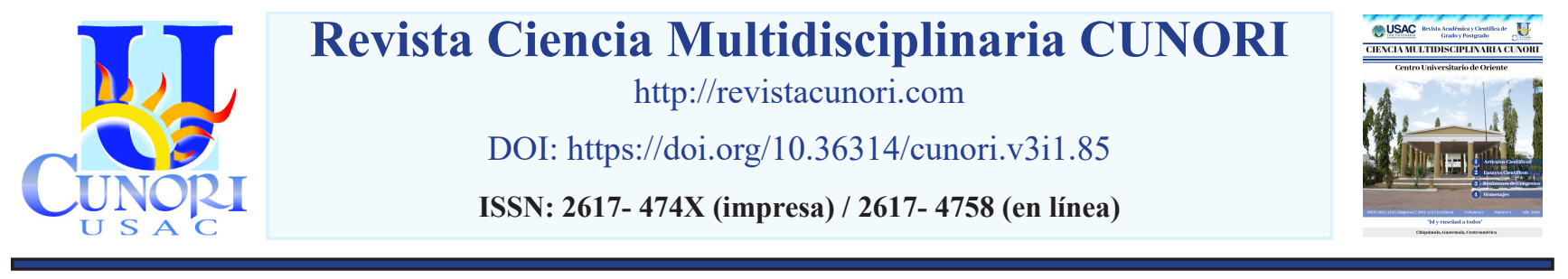

Como citar el artículo

Lemus, A., Vides, R., Paiz, J., Arriola, C., Mazariegos, E., (2019). Trastornos del sueño en médicos. Revista Ciencia Multidisciplinaria CUNORI, 3(1), 101-107. DOI: https://doi.org/10.36314/cunori.v3i1.85

\title{
Trastornos del sueño en médicos
}

\section{Sleep disorders in physicians}

\author{
Aylwin Lemus*, Rory Vides, Judith Paiz, Carlos Arriola, Edvin Mazariegos \\ Centro Universitario de Oriente (CUNORI), Universidad de San Carlos de Guatemala
}

Recibido: 17 de septiembre de 2018 / Revisión: 28 de septiembre de 2018 / Aceptado: 22 de enero de 2019

Disponible en internet el 30 de agosto de 2019

*Autor para correspondencia.

Correo electrónico: obed@love.com

\section{Resumen}

$\mathrm{L}$ os trastornos del sueño constituyen un grupo muy heterogéneo de procesos que constituyen uno de los problemas de salud más relevantes, entre el 30-40\% de la población mundial sufre de trastornos como insomnio, 1-10\% padecen de apnea de sueño y aproximadamente el $60 \%$ de los trabajadores por turnos reportan alteraciones en el ritmo circadiano. Los médicos quienes trabajan por turnos nocturnos y tener necesidad de laborar son los más afectados (Carro, 2013:01). Aplicando el cuestionario trastornos de sueño Monterrey, para medir la prevalencia se realizaron tablas simples con análisis univariado de los 12 trastornos que mide el cuestionario. De los 40 médicos estudiados, 12.5\% (5/40) del Instituto Guatemalteco de Seguridad Social sede Chiquimula y 87.5\% (35/40) del Hospital Nacional de Chiquimula de los cuales el 47.5\% (19/40) presento trastornos del sueño. La prevalencia de los trastornos de sueño fue: ronquido $58 \%$ (11/19), somnolencia excesiva diurna con $37 \%$ (7/19), piernas inquietas con $21 \%$ (4/19), insomnio con 11\% (2/19), mientras que parálisis del sueño y bruxismo con 5\% (1/19) cada uno respectivamente. Se determinó que para el mes de junio de 2018 la prevalencia de los trastornos del sueño fue de 48 por cada 100 médicos que realizan turnos, se debe dar continuidad a este tipo de estudios u otros que permitan la evaluación regular a los profesionales de la salud sobre los trastornos del sueño, como también estudiar la calidad asistencial de los mismos, e incentivar actividades de promoción y fomento de la higiene del sueño.

Palabras clave: trastornos de sueño, médicos, turnos

\section{Abstract}

Cleep disorders constitute a very heterogeneous group of processes that constitute one of the most relevant health problems, between $30-40 \%$ of the world population suffer from disorders such as insomnia, 1-10\% suffer from sleep apnea and approximately $60 \%$ of shift workers report alterations in the circadian rhythm. Doctors who work night shifts and need to work are the most affected. Using the Monterrey sleep disorders questionnaire, simple tables were analyzed to measure prevalence with univariate analysis of the 12 disorders measured by the questionnaire. Of the 40 doctors studied, 12.5\% (5/40) of the Guatemalan Social Security Institute, Chiquimula, and 87.5\% (35/40) of the National Hospital of Chiquimula, of which 47.5\% (19/40) had sleep disorders. The prevalence of sleep disorders was: snoring 58\% (11/19), excessive daytime sleepiness with 37\% (7/19), restless legs with 21\% (4/19), insomnia with $11 \%(2 / 19)$, while sleep paralysis and bruxism with 5\% (1/19) each respectively. It was determined that for the month of June 2018 the prevalence of sleep disorders was 48 per 100 physicians who take turns, this type of studies should be continued or others that allow the regular assessment of health professionals on sleep disorders, as well as studying their quality of care, and encouraging activities to promote and promote sleep hygiene.

Keywords: sleep disorders, doctors, shifts 


\section{Introducción}

Los trastornos del sueño son un grupo heterogéneo de procesos, cuya patología es muy frecuente tanto aislada, propia per se, o asociada a otras patologías, de hecho, es difícil encontrar alguna enfermedad que no altere en nada el sueño nocturno o la tendencia a dormir durante el día (Gállego, 2007:20). Es importante señalar que los trastornos del sueño se han identificado como factor de riesgo importante en patologías como la hipertensión arterial, infarto agudo al miocardio entre otras (Araoz, 2011:29).

Los trastornos que afectan la capacidad para conciliar o mantener el sueño y que se caracterizan por un exceso de sueño o que originan conductas anómalas relacionadas con el mismo. De acuerdo con el reporte del Centro Nacional de Investigaciones de Trastornos del Sueño, más de 40 millones de estadounidenses sufren de alteraciones del sueño crónicas e insomnio. Cerca del 35\% tienen dificultades para iniciar o mantener el sueño, presentan despertares precoces o sueño no reparador y $10 \%$ refieren que esto es causa persistente de interferencia seria en sus actividades diarias.

Las cuatro principales y más frecuentes quejas relacionadas con el dormir de los pacientes y que los orillan a buscar atención médica son insomnio, somnolencia excesiva diurna, ronquido y movimientos o conductas anormales durante el sueño (Collado, 2016:88).

Empero un grupo de trabajadores que a pesar de estar muy relacionados con el tratamiento de los mismos no están exentos de padecer dichos trastornos ya que se ven obligados a estar disponibles las 24 horas para atender a las personas que requieren cuidados especializados. Esto implica que los trabajadores del sector salud estén siempre expuestos a largas jornadas laborales incluyendo turnos nocturnos (Barahona, 2016:27).

Debido a eso se cree que los médicos en el cumplimiento de sus deberes se ven sometidos a estrés y en ocasiones (dependiendo de la asignación contractual), a la realización de turnos nocturnos y/o que sobrepasan las horas recomendadas de labores; esto obviamente genera alteraciones en organismo y la falta de sueño trae con ella alteraciones que podrían desencadenar otras patologías (Nieves, 2015:16).

\section{Materiales y métodos}

Estudio descriptivo aplicando el cuestionario trastornos de sueño Monterrey, para medir la prevalencia se realizaron tablas simples con análisis univariado de los 12 trastornos que mide el cuestionario los cuales fueron analizados.

El cuestionario de trastornos de sueño Monterrey, evalúa los principales trastornos de sueño por medio de 30 ítems los cuales se califican según la escala de Likert donde puntuación 4-5 se tomaron para determinar la presentación de dicho trastorno. Para la calificación por subescalas del cuestionario y determinar cuál es el trastorno que presentaban se hizo tomando en consideración qué un puntaje igual o mayor al asignado en la casilla de 4 y 5 que correspondían a casi siempre y siempre respectivamente sirvió para diagnóstico del trastorno (Téllez et al, 2012:152). 
También se caracterizó a los médicos que padecían trastornos según rango de edad, sexo, estado civil, residencia, horas del turno, ciclo de los turnos, grado académico, horas de trabajo al día, cuya información se obtuvo en la entrevista.

\section{Resultados}

Pacientes con trastorno de sueño

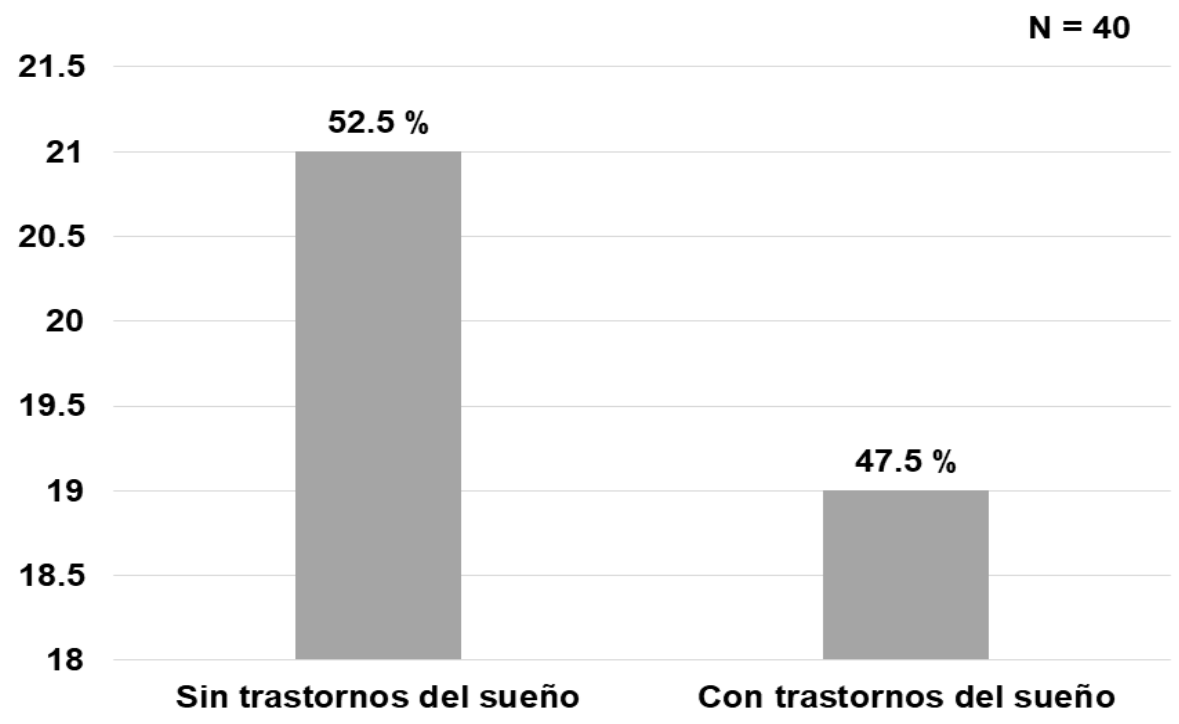

Figura 1. Distribución de pacientes con trastornos del sueño según el cuestionario de trastornos de sueño Monterrey, en el Hospital Nacional de Chiquimula e Instituto Guatemalteco de Seguridad Social de Chiquimula durante el mes de junio de 2018.

Se demuestra que la presencia de algunos de los trastornos del sueño de forma global fue del 47.5\%, mientras que el 52.5\% no presento algún síntoma asociado a los mismos de entre los médicos entrevistados. 


\section{Distribución de los trastornos del sueño}

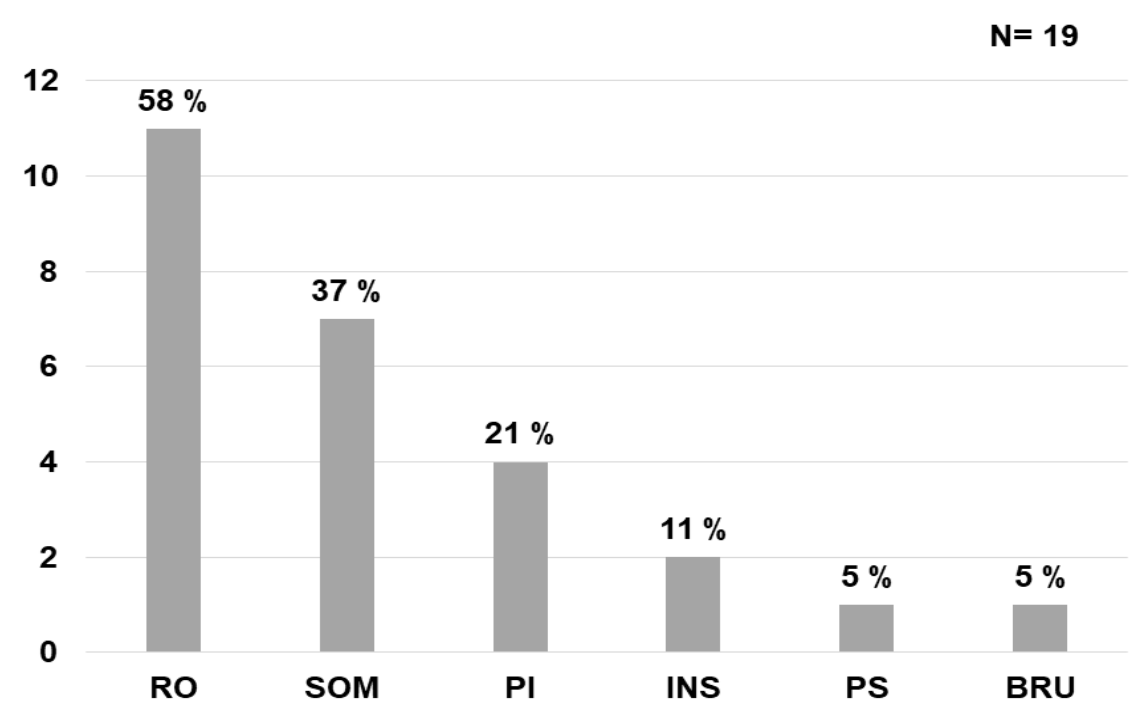

Figura 2. Distribución de los trastornos del sueño en médicos del Hospital Nacional de Chiquimula e Instituto Guatemalteco de Seguridad Social de Chiquimula en junio de 2018.

Tras la valoración con el cuestionario de Monterrey, se encontró que los trastornos del sueño mas frecuentes en la población medica fueron; roncar (RO) con 58\% (11), somnolencia excesiva diurna (SOM) con 37\% (7), piernas inquietas (PI) con 21\% (4), insomnio (INS) con 11\% (2), mientras que parálisis del sueño (PS) y bruxismo (BRU) con 5\% (1) cada uno respectivamente.

Se determinó que los médicos que presentaron algún trastorno del sueño son los de rango de edad de 27 a 34 años con el 63\% de los casos, siendo los del sexo masculino, casados los más afectados con un 58\% y un $63 \%$ respectivamente, y que residen en Chiquimula con un 68\%. Se determinó así mismo que los que poseen una maestría como grado académico tienen mayor presencia de trastornos del sueño con el $63 \%$ de los médicos, siendo esta la ginecología y obstetricia la especialidad que más reportes de casos tiene con un $41 \%$. En relación a la duración del turno, ciclos de los turnos y horas diarias trabajadas presentaron una prevalencia de $84 \%$ los que su turno duraba 16 horas, un $73 \%$ los que turnan cada 6 días, y de $37 \%$ los que trabajan más de 12 horas diarias.

\section{Discusión}

En el estudio se encontró que la presencia de algunos de los trastornos del sueño de forma global fue del 47.5\%, mientras que el 52.5\% no presento algún síntoma asociado a los mismos de entre los médicos entrevistados. Así mismo presentó que el trastorno de sueño más frecuente fue los ronquidos o roncar con una prevalencia del 58\% muy similar al resultado encontrado por Téllez y colaboradores en otro estudio en el cual el $60 \%$ presentaban dicho trastorno mientras duermen. seguido de la somnolencia excesiva diurna con $37 \%$ de los estudiados. Sin embargo, en relación con la prevalencia de síntomas de piernas inquietas, los resultados del estudio de Téllez et al que reporta una prevalencia de $24.6 \%$ son muy semejantes al valor encontrado, que presentó una prevalencia de $21 \%$ cuyos resultados son consistentes con la literatura investigada que reporta alta frecuencia del mismo. 
Otro trastorno que mostró moderada frecuencia en este estudio es el insomnio con un $11 \%$ de los casos estudiados, que es muy similar a la obtenida por Téllez et al. De 10 a $15 \%$ de la población en general. En otro sentido, los trastornos menos frecuentes en este estudio fueron: bruxismo y parálisis del sueño, lo cual es similar a lo encontrado en otras investigaciones según Téllez, con 5\% cada una respectivamente.

El rango de edad de los médicos que padecen de algún trastorno del sueño va de 27 a 34 años que representó el 63\% siendo el de mayor casos, seguido de 35 a 42 años quienes ocupan un segundo lugar con el $21 \%$ de los médicos que padecen de este tipo de trastornos, sin embargo en literatura revisada hay datos que revelan que la población adulta sobre todo en edades mayores de 45 años la prevalencia de los trastornos del sueño según Téllez, aunque en el estudio se demostró que el rango de edad con la menor prevalencia fue de 43 a 50 años con solo el 5\% de los médicos en el estudio.

Con respecto al sexo que predomina con trastornos del sueño es el género masculino con un $58 \%$ mientras que el género femenino obtuvo un $42 \%$, en contra parte con otros estudios como los de Carro, Alfaro y Boyano, quienes reportan que la mayor prevalencia del sueño la poseen las mujeres. Los resultados obtenidos demuestran que los médicos que padecen de trastornos del sueño son los médicos de Chiquimula con un 63\%; seguidos de los procedentes de Guatemala y Zacapa con $11 \%$, quienes mostraron baja prevalencia de los trastornos fueron los de Jocotán y Jutiapa quienes comparten solo 5\% cada uno respectivamente.

Con respecto al estado civil de los médicos que padecen trastornos del sueño prevalecen los casados con $63 \%$ de los mismos, en comparación con un 37 de los solteros, la importancia de conocer estos datos se debe a los problemas que estos trastornos conllevan a los matrimonios, como los reportados por Estivill y Rodríguez, quienes expresan en su estudio, que las personas con trastornos del sueño se divorcian 3 veces más que los que no padecen trastornos del sueño.

Misma representación según el grado académico que posee cada médico que presentó trastornos del sueño según el cuestionario de Monterrey, quedando de la siguiente manera quiénes poseen maestría con $63 \%$, mientras que los que sólo cuentan con la licenciatura un $37 \%$, lo que evidencia que la maestría representa algún grado de riesgo para padecer dichos trastornos. De los médicos que poseen maestría la especialidad con más frecuencia de los trastornos fue ginecología y obstetricia con $41 \%$, muy cercano a los valores encontrados en estudios previos como en el de López, seguido de cirugía con un 25\%, por el contrario, en este estudio las especialidades de medicina interna y pediatría comparten mismo porcentaje con $17 \%$ respectivamente.

Otra característica importante que se encontró en este estudio es que los médicos qué padecen trastornos del sueño laboran más de 12 horas al día con un $37 \%$ de los médicos que participaron en el presente estudio, seguido por los que laboran 8 horas al día con $32 \%$, por último, quienes presentaron frecuencia importante en los trastornos del sueño son los que laboran 4 horas al día con un $21 \%$. Se encontró, además, que los médicos que laboran en turnos nocturnos que son de 16 horas tienen una prevalencia de $84 \%$ de los trastornos del sueño, mientras que los que turnan las 24 horas, solo el 16\% presentó algún trastorno del sueño, todos datos muy semejantes encontrados por Diaz y colaboradores en un estudio en personal sanitario, el cual demostró que la prevalencia de los trastornos del sueño era similar a la de la población general, porque las turnos no parecían asociarse a dichos trastornos. 
Como se puede observar los trastornos del sueño en la población médica estudiada, se encuentran entre los valores de los estudios citados, esta situación es de prever al tratarse de una población expuesta a factores propios de su profesión, como lo es el ciclo de cada turno donde los médicos que turnan cada 6 días, representan el 73\% de los que mostraron algún trastorno del sueño, seguido de los que turnan cada 3 días quienes representan el 16\% de los que padecen dichos trastornos y en una escala menor como lo es el $11 \%$ quienes turnan cada 5 días.

\section{Referencias bibliográficas}

Araoz, R., Virhuez, Yblin., \& Guzmán, H. (2011). Síndrome de Apnea Obstructiva del Sueño como factor de riesgo para otras enfermedades. Revista Científica Ciencia Médica, 14(1), 25-30. Recuperado en 06 de septiembre de 2018, de http://www.scielo.org.bo/scielo. php?script=sci_arttext\&pid=S1817-74332011000100008\&lng=es\&tlng=es.

Barahon, A., Vidaurre, E., Sevilla, F., Rodríguez, J. \& Monge, S. (2013). El trabajo nocturno y sus implicaciones en la salud de medicos, enfermeras y oficiales de Medicina Legal de Costa Rica 30(1):17-36. Disponible en http://www.scielo.sa.cr/scielo.php?script=sci_arttext\&pid=S140900152013000100003

Carro García, T; Alfaro Acha, A; Boyano Sánchez, I. 2006. Trastornos del sueño, Tratado de geriatría. España, SEGG. Disponible en https://www.segg.es/ tratadogeriatria/PDF/S35-05\%2026_II.pdf

Collado, M. et al. (2016). Epidemiologia de los trastornos del sueño en población mexicana: seis años de experiencia en un centro de tercer nivel. Anales Médicos 61(2):87-92. Disponible en http://www.medigraphic.com/pdfs/abc/bc-2016/bc162b.pdf

Gállego, J., Toledo, J., Urrestarazu, E., Iriarte, J. (2007). Clasificación de los trastornos del sueño. Anales del Sistema Sanitario de Navarra 30 (supl.1):19-36. https://doi.org/10.4321/ S1137-66272007000200003

Nieves, D., Ordoñez M. \& Campoverde, M. (2015). Prevalencia y factores asociados a trastornos de sueño en personal de la salud en el Hospital Vicente Corral Moscoso, 2014. Cauca, Ecuador, Universidad de Cuenca. p. 86. Disponible en http://dspace.ucuenca.edu.ec/handle/ 123456789/21427

Tellez, A., Villegas, D., Juarez, D. \& Segura, G. (2012). Cuestionario de trastornos del sueño Monterrey. Medicina Universitaria 14(56):150-156. Disponible en http://www.elsevier.es/es-revista-medicina-universitaria-304-articulo-cuestionario-trastornos-del-sueno-monterrey-X1665579612676640

\section{Sobre el autor}

\section{Aylwin Obed Lemus Lemus}

Médico y cirujano, egresado del Centro Universitario de Oriente CUNORI, de la Universidad de San Carlos de Guatemala. Ha realizado las siguientes investigaciones: Conocimientos, actitudes y practicas sobre diferentes métodos anticonceptivos y caracterización de las pacientes post parto. Evaluación de las cuatro maniobras de Leopold en el CUNORI. Reanimación cardiopulmonar básica y avanzada en pediatría. La más reciente Trastornos del sueño en médicos. 


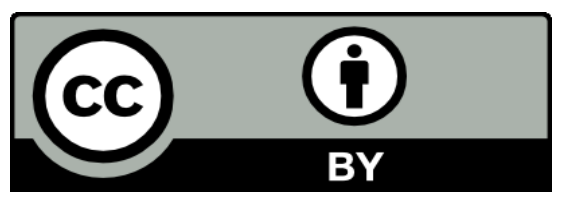

Este texto está protegido por una licencia CreativeCommons 4.0.

Usted es libre para compartir, copiar y redistribuir el material en cualquier medio o formato y adaptar el documento, remezclar, transformar y crear a partir del material para cualquier propósito, incluso comercialmente, siempre que cumpla la condición de atribución: usted debe reconocer el crédito de una obra de manera adecuada, proporcionar un enlace a la licencia, e indicar si se han realizado cambios. Puede hacerlo en cualquier forma razonable, pero no de forma tal que sugiera que tiene el apoyo del licenciante o lo recibe por el uso que hace. 